\title{
Onto Computing the Uncertainty for the Odometry Pose Estimate of a Mobile Robot
}

\author{
Josep M. Mirats Tur \\ Institut de Robòtica i Informàtica Industrial \\ CSIC-UPC \\ C/Llorens i Artigas, 4-6, 08028, Barcelona, Spain \\ jmirats@iri.upc.edu
}

\begin{abstract}
Solving the navigation issue for a mobile robot in a $2 D$ space requires using internal and external sensors, so researchers try to fuse data from different sensors using methods as for example Kalman filtering. Those methods need an estimation of the uncertainty in the pose estimates obtained from the sensory system, usually expressed by a covariance matrix and obtained from experimental data. In a previous work, a general method to obtain the uncertainty in the odometry pose estimate was proposed. Here, with the aim of assessing the generality of the method, the general formulation is particularized for a given differential driven robot. Its kinematic model relates two internal measurements: the instantaneous displacement of both, right and left wheels. The obtained formulation is validated experimentally and compared against Kalman filtering.
\end{abstract}

\section{Introduction}

Providing whether a robot or an autonomous vehicle (AV), with the ability to satisfactory perform a general task while robustly navigating in the real world, is a very difficult problem that, nowadays, has not been solved yet by the scientific community [1]. To reliably perform a task it is necessary to locate those vehicles, to know their pose $^{1}$ as well as its associated uncertainty, i.e., knowing both, where the vehicle is and how much the error on this pose estimate is. Usually, to accurately provide a pose estimate, a unique source of pose information is not enough, so researchers try to fuse data from different sources [2-5]. When fusing information, Kalman filtering is frequently used with the problem of determining the covariance matrices associated with the position uncertainty, since the errors of the sensory system affects the pose estimate. These matrices are determined experimentally from sensor data with different algorithms which trend to be very sensitive to

\footnotetext{
${ }^{1}$ Pose is used to denote the state vector formed by the robot's $2 \mathrm{D}$ position $(\mathrm{x}, \mathrm{y})$ plus its heading $(\theta)$
}

this parameter determination and initialization. Furthermore, general and unconstrained motion is usually assumed for the computation of those matrices, hence ignoring the nature of phenomena as, for instance, when the movement is constrained by non-holonomic architecture. This fact leads to poor pose uncertainty estimation.

Different methods have been previously proposed in the literature to estimate the pose uncertainty. For example, [6] employed a min/max error bound approach, [7] used a scalar as position uncertainty measurement, without reference to the orientation error. Uncertainty expressed by a covariance matrix is used in many works as [8-12]. In [13] a method for determining this covariance matrix is given for the special case of circular arc motion with constant radius of curvature. In [14] the odometry error is modelled for a synchronous drive system depending on parameters characterizing both systematic and non-systematic components. In a recent published research effort [15] a general method was proposed to compute the uncertainty in the pose estimate obtained from internal sensors, and applied to a particular non-holonomic, Ackerman driven, AV. The pose uncertainty is expressed using a covariance matrix and determined from the pose estimation equations given by the robot's kinematic model. It remained to assess that the proposed method is valid for any platform and sensory system used. In this paper the particularization of the general formulation given in [15] for the special case of a differential driven robot is presented.

Next sections are devoted to the general description of the method and its application to a particular robot. For this vehicle, a brief description of its kinematic model and the required mathematical development to derive the expression for the covariance matrix representing the pose estimate uncertainty are given. Finally, the herein proposed method has been compared against the standard Extended Kalman Filter (EKF) when computing the uncertainty associated with the odometry pose estimate. 


\section{Obtaining the pose estimate uncertainty}

Consider a mobile vehicle for which a kinematic model of the form $M(x, y, \Delta r, s, t)$ is given. Model $M$ is generally non-linear on $x, y, \theta$, (pose of the vehicle on the plane), $r$ (physical parameters of the vehicle), vector $s$ (internal sensor measurements), and $t$ (discrete time moments). We are concerned with the problem of accurately determining the uncertainty associated with the robot's pose computed from its internal sensors using the kinematic model.

Suppose that for time $t-1$ (notation $t-1$, for $t-\Delta t, t-2$ for $t-2 \Delta t$, and so on it is being used all throughout the paper) the pose, $P(t-1)=[x(t-1), y(t-1), \theta(t-1)]$, of the vehicle and its associated uncertainty, $\operatorname{Cov}\left[P_{t-1}\right]$, are known. For time $t$, after the vehicle has performed a certain movement, and sensors on the robot have noisily measured it, the new pose can be obtained using the equation $P(t)=P(t-1)+\Delta P(t)$. The pose increment will be computed from the kinematic model and actual sensor measurements. At this point, temporal variations on the physical parameters of the robot, and different sensor measurements for $\Delta x, \Delta y$ and $\Delta \theta$ are allowed so as to maintain generality in the model. $f, g, h$ represent general functions that model these increments for components $x$, $y$, $\theta$, respectively.

$$
\Delta P_{t}=\left(\begin{array}{c}
\Delta x_{t} \\
\Delta y_{t} \\
\Delta \theta_{t}
\end{array}\right)=\left(\begin{array}{l}
f(s(t), r(t), x(t-1), y(t-1), \theta(t-1)) \\
g(s(t), r(t), x(t-1), y(t-1), \theta(t-1)) \\
h(s(t), r(t), x(t-1), y(t-1), \theta(t-1))
\end{array}\right)
$$

The uncertainty in the robot's pose will depend on model inaccuracies, noise in the sensor measurements and additive errors from the previous pose estimate. It can be computed from the covariance matrix of the robot's pose:

$$
\begin{aligned}
\operatorname{Cov}\left[P_{t}\right]=\operatorname{Cov}\left[P_{t-1}+\Delta P_{t}\right]=\operatorname{Cov} & {\left[P_{t-1}\right]+\operatorname{Cov}\left[\Delta P_{t}\right]+} \\
& +\operatorname{Cov}\left[P_{t-1}, \Delta P_{t}\right]+\operatorname{Cov}\left[\Delta P_{t}, P_{t-1}\right]
\end{aligned}
$$

The term $\operatorname{Cov}\left[P_{t-1}\right]$ is recursive which can be initialized to $O_{3 \times 3}$ if the initial pose of the robot is well known. The term for the pose increment is $\operatorname{Cov}\left[\Delta P_{t}\right]$ and computed from the covariance definition as:

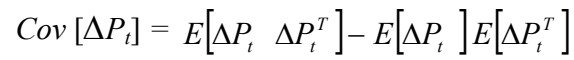

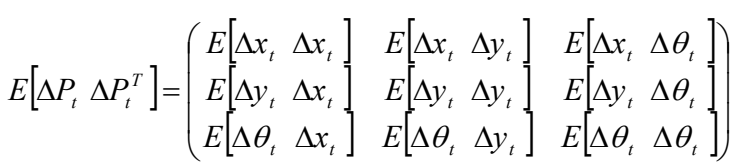

$$
\begin{aligned}
& E\left[\Delta P_{t}\right] E\left[\Delta P_{t}^{T}\right]=\left(\begin{array}{lll}
E\left[\Delta x_{t}\right] E\left[\Delta x_{t}\right] & E\left[\Delta x_{t}\right] E\left[\Delta y_{t}\right] & E\left[\Delta x_{t}\right] E\left[\Delta \theta_{t}\right. \\
E\left[\Delta y_{t}\right] E\left[\Delta x_{t}\right] & E\left[\Delta y_{t}\right] E\left[\Delta y_{t}\right] & E\left[\Delta y_{t}\right] E\left[\Delta \theta_{t}\right] \\
E\left[\Delta \theta_{t}\right] E\left[\Delta x_{t}\right] & E\left[\Delta \theta_{t}\right] E\left[\Delta y_{t}\right] & E\left[\Delta \theta_{t}\right] E\left[\Delta \theta_{t}\right]
\end{array}\right)(5)
\end{aligned}
$$

The cross terms $\operatorname{Cov}\left[P_{t-1}, \Delta P_{t}\right]+\operatorname{Cov}\left[\Delta P_{t}, P_{t-1}\right]$ express the influence of the previous position $P_{t-1}$, on the increment of run path $\Delta P_{t}$, mainly due to the dependence of $\Delta P_{t}$ in $\theta_{t-1}$. Computation of these terms requires complex mathematical expressions that will not add a deeper insight on the current study; for further information on how to compute them, please refer to [17].

So, equations (3) to (5) express the uncertainty of the pose determined from internal sensors while taking into account the physical architecture of the vehicle ${ }^{2}$. In order to solve (4) and (5), a distribution for the sensors noise must be assumed, which will be determined from experimental characterization.

\section{Used platform in the study}

A standard Pioneer P3 AT robot named Helena (see Fig. 1) has been used in this study. The vehicle is electrically powered and it can perform tasks autonomously. Used internal sensors are, for the case at hand, two odometers attached to the shafts of the right and left drive wheel motors respectively.

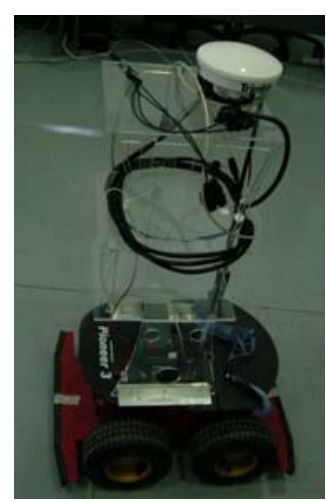

\section{Figure 1. Pioneer P3 AT robot Helena used in this study.}

The used driving architecture is differential. The kinematic model being used can be derived from Fig. 2 or found in any basic robotics book as [18]. It is not the scope of this paper to study kinematic models and/or how they are derived. Equation (6) expresses the kinematic model used for this study, where parameter $b$ is the robot width and $\left(V_{r}, V_{l}\right)$ denotes the right and left linear wheel control velocities respectively. The simple proposed model has the essential elements for the analysis and should be enough for control purposes.

\footnotetext{
2 Although $(x, y, \theta)$, are scalars, the Transpose is used in the formulation in order to be mathematically rigorous and to maintain generality.
} 


$$
\left(\begin{array}{l}
\dot{x} \\
\dot{y} \\
\dot{\theta}
\end{array}\right)=\left(\begin{array}{ll}
\frac{1}{2} \cos \theta & \frac{1}{2} \cos \theta \\
\frac{1}{2} \sin \theta & \frac{1}{2} \sin \theta \\
\frac{1}{b} & -\frac{1}{b}
\end{array}\right)\left(\begin{array}{l}
V_{r} \\
V_{l}
\end{array}\right)
$$

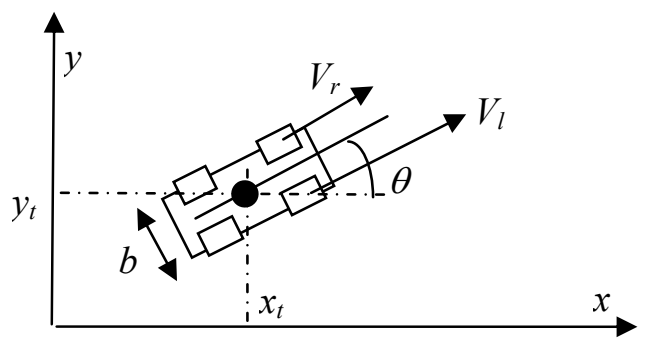

\section{Figure 2. Schematic draw for obtaining the kinematic model of a common differential robot.}

\section{Robot's pose estimate uncertainty.}

In order to assess the generality of the method given in [15], it is proposed to obtain the covariance matrix expressing the uncertainty for the odometry estimated pose from the equations of Helena's kinematic model. This uncertainty estimation takes directly into account the physical structure of the vehicle, which is translated into the control variables being manipulated and sensed. For the case at hand, the only inputs to the model are the two measurements being obtained from internal sensors: the instantaneous displacement for each of the robot wheels $\Delta d_{t}^{\text {right }}$ and $\Delta d_{t}^{\text {left }}$. The pose estimate from odometry at time $t$ is computed as, $P_{t}^{o d}=P_{t-1}+\Delta P_{t}^{o d}$, corresponding to the sum of the robot pose in a previous time $t-1$, plus the increments in pose measured from the odometry system and computed using the equations from the kinematic model.

$$
\Delta P_{t}^{\text {od }}=\left(\begin{array}{c}
\Delta x_{t}^{\text {od }} \\
\Delta y_{t}^{\text {od }} \\
\Delta \theta_{t}^{\text {od }}
\end{array}\right)=\left(\begin{array}{c}
\frac{\Delta \hat{d}_{t}^{\text {right }}+\Delta \hat{d}_{t}^{\text {left }}}{2} \cos \left(\theta_{t-1}\right) \\
\frac{\Delta \hat{d}_{t}^{\text {right }}+\Delta \hat{d}_{t}^{\text {left }}}{2} \sin \left(\theta_{t-1}\right) \\
\frac{\Delta \hat{d}_{t}^{\text {right }}-\Delta \hat{d}_{t}^{\text {left }}}{b}
\end{array}\right)
$$

Measurements obtained from both odometer sensors are not error free. In order to compute the uncertainty in the pose given by the odometry system, $\operatorname{Cov}\left[P_{t}^{\text {od }}\right]$, sensor errors are considered to follow a normal distribution:

$$
\Delta \hat{d}_{t}^{\text {right }}=\Delta d_{t}^{\text {right }}+\varepsilon^{\text {right }} \text { where } \quad \varepsilon^{\text {right }} \approx N\left(0, \sigma_{\text {right }}^{2}\right)
$$

$$
\Delta \hat{d}_{t}^{\text {left }}=\Delta d_{t}^{\text {left }}+\varepsilon^{\text {left }} \text { where } \quad \varepsilon^{\text {left }} \approx N\left(0, \sigma_{\text {left }}^{2}\right)
$$

So the uncertainty in the pose $P_{t}^{o d}$ is given by:

$\operatorname{Cov}\left[P_{t}^{o d}\right]=\operatorname{Cov}\left[P_{t-1}+\Delta P_{t}^{o d}\right]=\operatorname{Cov}\left[P_{t-1}\right]+$ $\operatorname{Cov}\left[\Delta P_{t}^{o d}\right]+\operatorname{Cov}\left[P_{t-1}, \Delta P_{t}^{o d}\right]+\operatorname{Cov}\left[\Delta P_{t}^{o d} . . P_{t-1}\right]$

The term $\operatorname{Cov}\left[P_{t-1}\right]$ is recursive and initialized to $0_{3 x 3}$ because the initial pose of the robot is supposed to be well known. For this particular study, and without lack of generality, it will be considered $\operatorname{Cov}\left[P_{t-1}, \Delta P_{t}\right]=\operatorname{Cov}$ $\left[\Delta P_{t}, P_{t-1}\right]=0$. In order to solve (3) for the used vehicle, it is necessary to calculate each of the elements of the involved matrices. The first element in equation (4) is calculated as follows:

$$
\begin{gathered}
E\left[\Delta x_{t}^{o d} \Delta x_{t}^{\text {od }}{ }^{T}\right]=E\left[\frac{\Delta \hat{d}_{t}^{\text {right }}+\Delta \hat{d}_{t}^{\text {left }}}{2} \cos \left(\theta_{t-1}\right) \frac{\Delta \hat{d}_{t}^{\text {right }}+\Delta \hat{d}_{t}^{\text {left }}}{2} \cos \left(\theta_{t-1}\right)\right] \\
=E\left[\left(\frac{\Delta \hat{d}_{t}^{\text {right }}+\Delta \hat{d}_{t}^{\text {left }}}{2} \cos \left(\theta_{t-1}\right)\right)^{2}\right] \\
=\frac{1}{4} \cos ^{2}\left(\theta_{t-1}\right) E\left[\left(\Delta d_{t}^{\text {right }}+\varepsilon^{\text {right }}+\Delta d_{t}^{\text {left }}+\varepsilon^{\text {left }}\right)^{2}\right] \\
=\cdots=\frac{1}{4} \cos ^{2}\left(\theta_{t-1}\right)\left\{\left(\Delta d_{t}^{\text {right }}\right)^{2}+\left(\Delta d_{t}^{\text {left }}\right)^{2}+2 \Delta d_{t}^{\text {right }} \Delta d_{t}^{\text {left }}+\sigma_{\text {right }}^{2}+\sigma_{\text {left }}^{2}\right\}
\end{gathered}
$$

Two main assumptions have been made here. First the orientation of the robot for time $t-1$ is considered as deterministic and without error. A deeper development could be made considering an error for this angle [17], expressed in the form $\theta_{t-1}+\varepsilon_{t-1}^{\theta}$, but it does not add new information to the process of showing the advocated method. Second, measurements from both odometers have been considered independent.

After computing all the expectations an expression for $\operatorname{Cov}\left[\Delta P_{t}^{\text {od }}\right]$ is obtained, as the rest of two symmetric $3 x 3$ matrices, $E\left[\Delta P_{t}^{o d} \Delta P^{T^{o d}}\right]-E\left[\Delta P_{t}^{o d}\right] E\left[\Delta P_{t}^{T^{o d}}\right]$. Defining the terms $k_{1}$ to $k_{5}$ as follows:

$$
\begin{gathered}
k_{1}=\left(\Delta d_{t}^{\text {right }}\right)^{2}+\left(\Delta d_{t}^{\text {left }}\right)^{2} \\
k_{2}=\left(\Delta d_{t}^{\text {right }}\right)^{2}-\left(\Delta d_{t}^{\text {left }}\right)^{2} \\
k_{3}=\Delta d_{t}^{\text {right }} \Delta d_{t}^{\text {left }} \\
k_{4}=\sigma_{\text {right }}^{2}+\sigma_{\text {left }}^{2} \\
k_{5}=\sigma_{\text {right }}^{2}-\sigma_{\text {left }}^{2}
\end{gathered}
$$

Elements for the first matrix, given in (3), are:

$$
\begin{gathered}
c_{11}=\frac{k_{1}+2 k_{3}+k_{4}}{4} \cos ^{2}\left(\theta_{t-1}\right) \\
c_{12}=c_{21}=\frac{k_{1}+2 k_{3}+k_{4}}{4} \cos \left(\theta_{t-1}\right) \sin \left(\theta_{t-1}\right)
\end{gathered}
$$




$$
\begin{gathered}
c_{13}=c_{31}=\frac{k_{2}+k_{5}}{2 b} \cos \left(\theta_{t-1}\right) \\
c_{22}=\frac{k_{1}+2 k_{3}+k_{4}}{4} \sin ^{2}\left(\theta_{t-1}\right) \\
c_{23}=c_{32}=\frac{k_{2}+k_{5}}{2 b} \sin \left(\theta_{t-1}\right) \\
c_{33}=\frac{k_{1}-2 k_{3}+k_{5}}{b^{2}}
\end{gathered}
$$

A complete development of all the elements for matrix (4) is given in the Appendix. The second matrix, (5), is computed considering the product of two vectors $E\left[\Delta P_{t}^{o d}\right] E\left[\Delta P_{t}^{T_{t}^{o d}}\right]$, where,

$$
E\left[\Delta P_{t}^{o d}\right]=\left(\begin{array}{c}
\frac{\Delta d_{t}^{\text {right }}+\Delta d_{t}^{\text {left }}}{2} \cos \left(\theta_{t-1}\right) \\
\frac{\Delta d_{t}^{\text {right }}+\Delta d_{t}^{\text {left }}}{2} \sin \left(\theta_{t-1}\right) \\
\frac{\Delta d_{t}^{\text {right }}-\Delta d_{t}^{\text {left }}}{b}
\end{array}\right)
$$

The full expression for this second matrix is not included, It can be easily and directly derived from multiplying $E\left[\Delta P_{t}^{\text {od }}\right] E\left[\Delta P_{t}^{T_{t}^{\text {od }}}\right]$. Finally, the expression for the uncertainty in the odometry pose estimate will be given by subtracting the two computed matrices: $E\left[\Delta P_{t}^{o d} \Delta P_{t}^{T_{t}^{o d}}\right]-E\left[\Delta P_{t}^{o d}\right] E\left[\Delta P_{t}^{T_{t}^{o d}}\right]$.

\section{Assessment of results}

To assess the effectiveness of the proposed method to compute the uncertainty associated with the odometry pose estimate of a mobile vehicle, an experiment was designed and run using the robot previously described in section III. A $10 \times 4.5 \mathrm{~m}$. rectangular path, marked on the floor of the Institute corridor, was followed while manually driving the described robot. Data from internal sensors (right and left encoders) were gathered during the run ${ }^{3}$. Figure 3 shows the obtained odometry data for coordinates $(x, y)$, being the horizontal axis $x$ and the vertical $y$. Then, the uncertainty of the odometry position estimates obtained with, both, an EKF and the proposed method was compared. Figures 4 and 5 show the obtained covariance for $P_{t}^{\text {od }}$, denoting the uncertainty of the position estimates, for the $x$ and $y$ axis using the proposed formulation; Figures 6 and 7 shows the obtained covariance in the position estimate $P_{t}^{\text {od }}$ using an EKF.

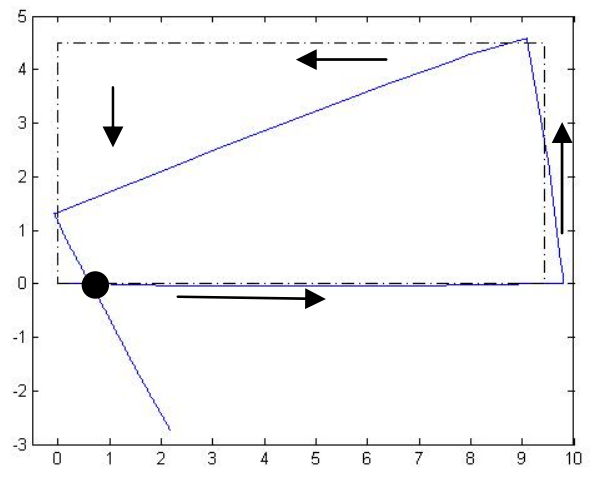

Figure 3. The path the robot computes from odometry data is shown in continuous line. The proposed path (depicted in dot-dashed line) starts at the origin $(0,0)$ advancing from left to right and from down to up, as shown by the arrows, in the graph to complete a round trip.

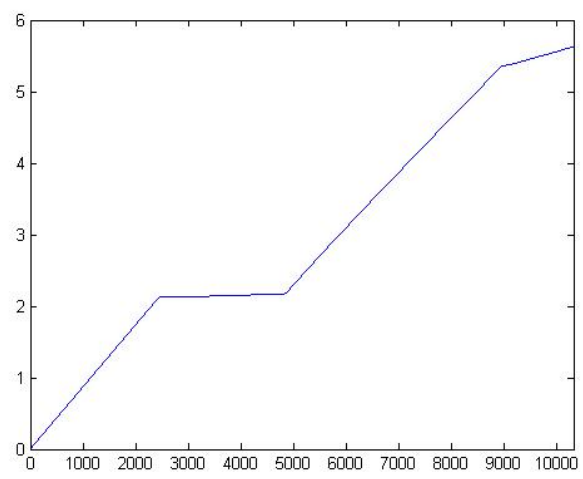

Figure 4. Estimated position uncertainty for coordinate $x$ (in the vertical axis) using the presented formulation.

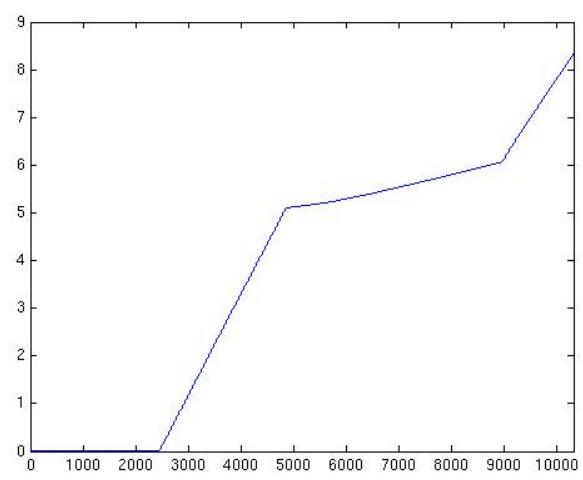

Figure 5. Estimated position uncertainty for coordinate $y$ (in the vertical axis) using the presented formulation.

\footnotetext{
${ }^{3}$ Maximum velocity was $20 \mathrm{~cm} / \mathrm{s}$. Sampling rate was $20 \mathrm{~Hz}$.
} 


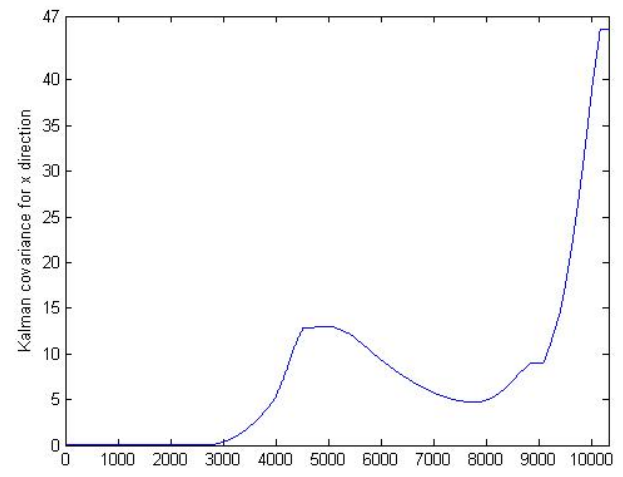

Figure 6. Estimated position uncertainty for coordinate $x$ (in the vertical axis) using an EKF.

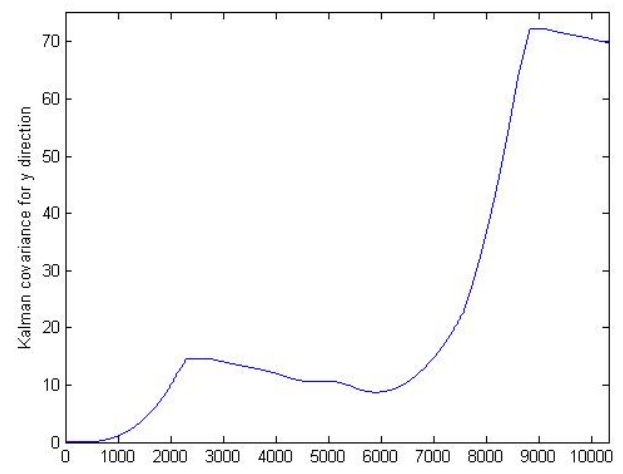

Figure 7. Estimated position uncertainty for coordinate $y$ (in the vertical axis) using an EKF.

It can be seen from figures 4 and 5 that the obtained uncertainty for coordinates $x, y$ using the proposed method (about 2.3 and 2.9 meters in the $x$ and $y$ axes respectively) is lower than the obtained with an EKF (about 6.8 and 8.4 meters in the $x$ and $y$ axes respectively). It turns out that the real measured position error for the used platform in the run experiment is about 2.2 and 2.75 meters for the $\mathrm{x}$ and $\mathrm{y}$ axes respectively (which means a covariance uncertainty of about 5 and 8 for $x$ and $y$ axes respectively, as the presented method accurately states).

Surprisingly, when using the EKF to estimate the pose uncertainty, a slight decrease for the position uncertainty in both axes is reported in figures 6 and 7 . This fluctuation is produced when the robot navigates in the opposite direction of the initial movement, that is, $\theta=180^{\circ}$. Author thinks it is produced by the effects of linearization of the system state transition equation. The elements of the obtained jacobians are trigonometric functions, $\sin (\theta)$ and $\cos (\theta)$, which causes the observed effect with the variation of theta, the robot's orientation. This is not fully consistent with the experiment run, because only one source of position information has been used to compute the position of the robot, so there is no extra information so as to decrease the uncertainty of the estimated position. In this sense, and at least for this experiment, the EKF correctly computed the pose of the robot, but failed to obtain its associated uncertainty.

On the other hand, the computed uncertainty with the presented method it does not depend on any parameter initialization as it is needed with the EKF, in this sense; we do not find variation of results depending on how the algorithm is initialized. It neither depends on the axis to which the robot is oriented. Obtained results are robust and consistent with the experiment run.

\section{Conclusions and future work}

The pose of a mobile robot in a two-dimensional space can be represented by the three components vector $(x, y, \theta)$ where $x, y$, represent the position coordinates of the robot on the space, and $\theta$ its heading orientation. Thus the robot pose at time $t$ is denoted as $P_{t}=\left(x_{t}, y_{t}\right.$, $\left.\theta_{t}\right)^{T}$. There is always an error associated with the movement of the autonomous platform. The imprecision in the pose estimates is due to errors in the sensory system used to determinate such estimations as well as unmodeled factors in the vehicle model. These errors are normally estimated from data derived from experimentation and then integrated in algorithms, as for example Kalman filtering, which are highly sensitive to the obtained parameters.

A method has been proposed to obtain an accurate expression for the pose uncertainty, by means of a covariance matrix, when position estimates are being obtained using internal sensors. The proposed method is valid for any platform or set of sensors. A particularization for the general formulation has been done here to obtain the covariance matrix representing the measurement of pose uncertainty for a given kind of differential driving robot.

Even if lot of research and experiments are left for a near future, the initial experimental evaluation demonstrates that the proposed method can compute the pose uncertainty with highest accuracy than the standard EKF method. It is desired in a near future the integration of such matrix computation into a probabilistic frame (errors in the positioning sensors can be assumed to be random and can be modeled by a parametric probability distribution) that allows performing data fusion from different positioning systems; concretely, work is being done on the integration of odometry, gyroscope, compass and GPS measurements. In a recent published work [17], we managed to fuse correlated GPS and odometry information. It is hoped that the formulation presented in this paper will help to reduce errors when estimating the pose of the vehicle using different sources of data. 


\section{Appendix}

A complete derivation of the terms of the first matrix expressed in (4) is given here. The first element in (4) was calculated in section IV. Taking into account that the final matrix is symmetric, the rest of elements are calculated as follows:

$$
\begin{gathered}
E\left[\Delta x_{t}^{\text {od }} \Delta y_{t}^{\text {od }}{ }^{T}\right]=E\left[\Delta y_{t}^{\text {od }} \Delta x_{t}^{\text {od }} T\right]= \\
=E\left[\left(\frac{\Delta \hat{d}_{t}^{\text {right }}+\Delta \hat{d}_{t}^{\text {left }}}{2}\right)^{2} \cos \left(\theta_{t-1}\right) \sin \left(\theta_{t-1}\right)\right] \\
=\frac{1}{4} \cos \left(\theta_{t-1}\right) \sin \left(\theta_{t-1}\right) E\left[\left(\Delta d_{t}^{\text {right }}+\varepsilon^{\text {right }}+\Delta d_{t}^{\text {left }}+\varepsilon^{\text {left }}\right)^{2}\right] \\
=\cdots=\frac{1}{4} \cos \left(\theta_{t-1}\right) \sin \left(\theta_{t-1}\right)\left\{\left(\Delta d_{t}^{\text {right }}\right)^{2}+\left(\Delta d_{t}^{\text {left }}\right)^{2}+2 \Delta d_{t}^{\text {right }} \Delta d_{t}^{\text {left }}+\sigma_{\text {right }}^{2}+\sigma_{\text {left }}^{2}\right\}
\end{gathered}
$$

Going on the calculation:

$$
\begin{aligned}
& E\left[\Delta y_{t}^{\text {od }} \Delta y_{t}^{\text {od }}{ }^{T}\right]=E\left[\left(\frac{\Delta \hat{d}_{t}^{\text {right }}+\Delta \hat{d}_{t}^{\text {left }}}{2} \sin \left(\theta_{t-1}\right)\right)^{2}\right] \\
& =\frac{1}{4} \sin ^{2}\left(\theta_{t-1}\right) E\left[\left(\Delta d_{t}^{\text {right }}+\varepsilon^{\text {right }}+\Delta d_{t}^{\text {left }}+\varepsilon^{\text {left }}\right)^{2}\right] \\
& =\cdots=\frac{1}{4} \sin ^{2}\left(\theta_{t-1}\right)\left\{\left(\Delta d_{t}^{\text {right }}\right)^{2}+\left(\Delta d_{t}^{\text {left }}\right)^{2}+2 \Delta d_{t}^{\text {right }} \Delta d_{t}^{\text {left }}+\sigma_{\text {right }}^{2}+\sigma_{\text {left }}^{2}\right\} \\
& E\left[\Delta x_{t}^{\text {od }} \Delta \theta_{t}^{\text {od }} T\right]=E\left[\Delta \theta_{t}^{\text {od }} \Delta x_{t}^{\text {od }} T\right]= \\
& =E\left[\left(\frac{\Delta \hat{d}_{t}^{\text {right }}+\Delta \hat{d}_{t}^{\text {left }}}{2}\right)\left(\frac{\Delta \hat{d}_{t}^{\text {right }}-\Delta \hat{d}_{t}^{\text {left }}}{b}\right) \cos \left(\theta_{t-1}\right)\right] \\
& =\cdots=\frac{1}{2 b} \cos \left(\theta_{t-1}\right)\left\{\left(\Delta d_{t}^{\text {right }}\right)^{2}-\left(\Delta d_{t}^{\text {left }}\right)^{2}+\sigma_{\text {right }}^{2}-\sigma_{\text {left }}^{2}\right\} \\
& E\left[\Delta y_{t}^{\text {od }} \Delta \theta_{t}^{\text {od }} T\right]=E\left[\Delta \theta_{t}^{\text {od }} \Delta y_{t}^{\text {od }} T\right]= \\
& =E\left[\left(\frac{\Delta \hat{d}_{t}^{\text {right }}+\Delta \hat{d}_{t}^{\text {left }}}{2}\right)\left(\frac{\Delta \hat{d}_{t}^{\text {right }}-\Delta \hat{d}_{t}^{\text {left }}}{b}\right) \sin \left(\theta_{t-1}\right)\right] \\
& =\cdots=\frac{1}{2 b} \sin \left(\theta_{t-1}\right)\left\{\left(\Delta d_{t}^{\text {right }}\right)^{2}-\left(\Delta d_{t}^{\text {left }}\right)^{2}+\sigma_{\text {right }}^{2}-\sigma_{\text {left }}^{2}\right\} \\
& E\left[\Delta \theta_{t}^{\text {od }} \Delta \theta_{t}^{\text {od }}{ }^{T}\right]=E\left[\left(\frac{\Delta \hat{d}_{t}^{\text {right }}-\Delta \hat{d}_{t}^{\text {left }}}{b}\right)^{2}\right] \\
& =\cdots=\frac{1}{b^{2}}\left\{\sigma_{\text {right }}^{2}-\sigma_{\text {left }}^{2}\right\}
\end{aligned}
$$

\section{References}

[1] H. Gonzalez-Banos and J. C. Latombe., "Navigation strategies for exploring indoor environments", Int. Journal of Robotics Research, 21(10-11), pp. 829-848, Oct-Nov. 2002.
[2] M.A. Abidi, R.C. González, "Data fusion in robotics and machine intelligence", Academic Press Professional, Inc., 1992, ISBN:0-12-042120-8.

[3] R.C. Luo, Chih-Chen Yih, Kuo Lan Su, "Multisensor fusion and integration: approaches, applications, and future research directions", IEEE Sensors Journal, 2(2), Apr. 2002, pp. 107-119.

[4] H. Chung, L. Ojeda, and J. Borenstein, "Sensor fusion for mobile robot dead reckoning with a precision-calibrated fiber obtic gyroscope", Proc., IEEE Int. Conference on Robotics and Automation (ICRA), Seoul, Korea, May 2001, pp. 3588-3593.

[5] S. Majumder, S. Scheding, H.F. Durrant-Whyte, "Multi sensor data fusion for underwater navigation", Robotics and Autonomous Systems, 35(1), pp. 97-108, 2001.

[6] R.A. Brooks, "Visual map making for a mobile robot", Proc., IEEE Int. Conference on Robotics and Automation (ICRA), St. Louis, Missouri, 1985, pp.824-829.

[7] R. Chatila, and J. P. Laumond, "Position referencing and consistent world modeling for mobile robots", Proc., IEEE Int. Conference on Robotics and Automation (ICRA), St. Louis, Missouri, 1985, pp.138-145.

[8] R.C. Smith, and P. Cheeseman, "On the representation and estimation of spatial uncertainty", Int. Journal of Robotics Research, 5, pp. 55-68, 1987.

[9] C.M. Wang, "Location estimation and uncertainty analysis for mobile robots", Int. Conference on Robotics and Automation (ICRA), 1988, pp. 1230-1235.

[10] A. Kelly, "General solution for linearized systematic error propagation in vehicle odometry," in Proc. Int. Conference Intelligent Robot and Systems (IROSO1), Maui, HI, Oct-Nov. 2001, pp. 1938-1945.

[11] A. Kelly, "Linearized error propagation in odometry", Int. Journal of Robotics Research, 23(2), Feb. 2004, pp. 179218.

[12] A. Pozo-Ruz, "Sistema sensorial para localización de vehículos en exteriores", PhD., Dept. Elect. Eng., University of Málaga, Spain, ISBN:84-699-8140-4, 2001.

[13] K.S. Chong and L.Kleeman, "Accurate odometry and error modeling for a mobile robot", Int. Conference on Robotics and Automation (ICRA), 1997, pp. 2783-2788.

[14] A. Martinelli, "The odometry error of a mobile robot with a synchronous drive system", Transactions on Robotics and Automation, Vol. 18, pp. 399-405, Jun. 2002.

[15] J.M. Mirats Tur, J.L. Gordillo and C. Albores Borja, "A Closed-Form Expression for the Uncertainty in Odometry Position Estimate of an Autonomous Vehicle", Transactions on Robotics, Vol. 21, No. 5, October 2005.

[16] C. Albores, "Analysis, Architecture, and Fusion Methods for Vehicle Automation", PhD, ITESM, Mexico, March 2007.

[17] J.M. Mirats Tur, C. Albores, "Outdoor robot navigation based on a probabilistic data fusion scheme", accepted at the IROS 2007 conference to be held in S. Diego.

[18] A. Ollero, "Robótica: manipuladores y robots móviles", Ed. Marcombo Boixareu, Barcelona, 2001. 